\title{
Multi-spectral Electron Microprobe - Now and the Future
}

\author{
Colin M. MacRae ${ }^{1}$, Nick C. Wilson ${ }^{1}$ and Aaron Torpy ${ }^{1}$ \\ ${ }^{1}$ Microbeam Laboratory, CSIRO Process Science and Engineering, Clayton, Australia 3169 \\ Corresponding author email: Colin.MacRae@csiro.au
}

Hyperspectral datasets are now routinely collected in electron microprobes during mapping with an increasing amount of the energy spectrum being covered. Energy dispersive spectrometers (EDS) are now giving spectral information on X-rays down to Li [1], with the development of soft X-ray emission spectroscopy (SXES) giving high resolution spectroscopy down to energies of $50 \mathrm{eV}$ [2], while cathodoluminescence (CL) detectors are giving spectral information in the ultra-violet to the infra-red range [3]. The SXES detector gives direct measurement of Si-L (100eV), Al-L (70eV) and importantly $\mathrm{Li}-\mathrm{K}(52 \mathrm{eV})$ using first order lines, while higher order $\mathrm{x}$-ray reflections can be used to probe other elements which do not have first order lines able to be directly measured using this spectrometer. The high energy resolution of the detector, down to $0.2 \mathrm{eV}$ [2], can differentiate many of the overlapping $\mathrm{x}$ ray lines observed using wavelength dispersive spectrometers, as well as resolve peak shape and energy changes which aid in the determination of the chemical bonding state of various compounds. CL also captures bonding and valence information having a high energy resolution over a comparatively small energy range which, depending on configuration, can be down to $10 \mathrm{meV}$. Both the SXES and CL techniques are capable of detecting trace element information down to the ppm in mapping mode.

Hyperspectral data collection enables spectra to be accumulated in a single mapping pass avoiding artifacts from beam damage, which can induce bond changes, charge trapping and elemental migration. This can lead to spectral shape changes in CL and SXES and elemental migration changes in x-ray emission. The ability to collect all these signals in parallel, generating multi-spectral data sets, in an electron microprobe equipped with a field emission gun offers a wealth of chemical state and valence information, elemental levels at detection limits of a few ppm together at analytical resolutions of down to hundreds of nanometers. Multi-spectral data sets have the great advantage of allowing post-hoc examination of the data. This can lead to the discovery of unexpected phases (in x-ray data), bonding changes (in the SXES data) and defects or centers (in the cathodoluminescence data) within a sample. All this information is available at the pixel level. A problem encountered when trying to examine hyperspectral datasets is how to process the data to extract key spectral signatures. There are several approaches, with popular automated methods being principal component analysis, which reduces the dimensionality of the data set [4], and data clustering [5]. This operation can be performed on all hyperspectral data in parallel or separately and then combined at the end where appropriate, Fig. 1. Separately processing the data sets can be required due to the different generation processes and detectors associated with them. These processing algorithms find not only the major phases present but can locate minor and rare phases, all of which can be quantified in the WDS and EDS cases, through the application of peak identification, peak fitting and matrix correction methods. Phases found in the SXES and CL data can be fitted to identify bonding, valance and potentially trace information. The aim of this paper is to discuss what additional information hyperspectral data analysis can yield for chemically and structurally complex minerals and materials.

\section{References:}

[1] L. Xiaobing et al, Microsc. Microanal. 19 (Suppl 2), 2013, p 1136-1137.

[2] M. Terauchi et al, Journal of Electron Microscopy. 61(1): (2012), p. 1-8. 
[3] C.M. MacRae et al, Microsc. Microanal. 18, (2012), p. 1239-1245.

[4] P.G. Kotula et al, Microsc. Microanal. 9, (2003), p. 1-17

[5] N.C. Wilson et al, Microsc. Microanal. 14(Suppl 2), (2008), 434-435CD.
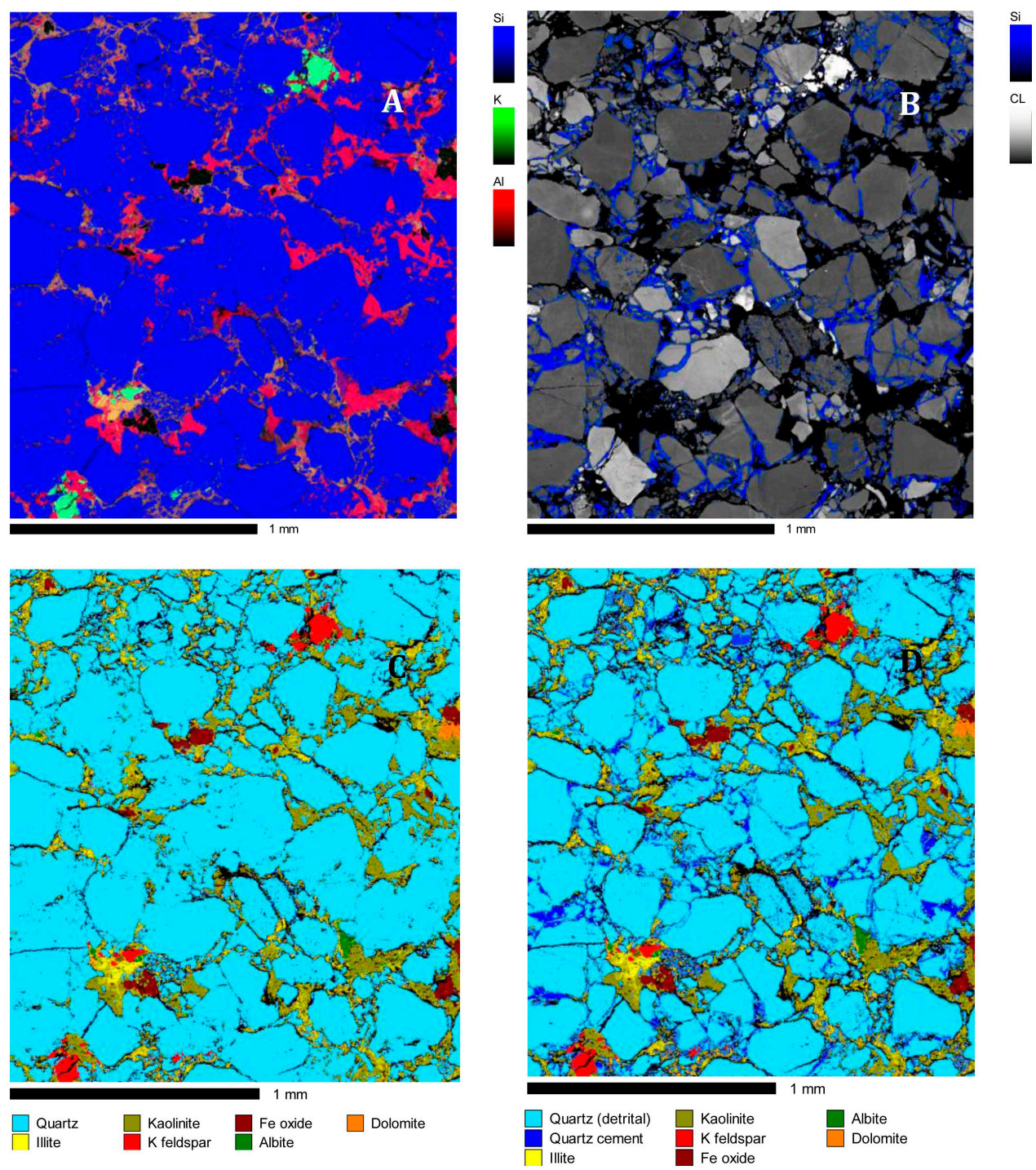

Figure 1. A. Sandstone sample showing detrital quartz intergrown with clays (Kaolinite, Illite and Muscovite). Quartz grain structure is no evident with x-rays. B. Grain structure of quartz and hydrothermal healing within quartz grains is evident in a combined cathodoluminescence and silicon map. Regions containing detrital quartz have been grayed to highlight hydrothermal quartz features. C. Clustered x-ray map showing main minerals present. D. Combined X-ray and cathodoluminescence clustering shows the fine scale hydrothermal quartz sealing the detrital quartz and "bonding" the mineral assemblage. 